\title{
The Research of the Model of the Limited Perception of the Virtual Soldier
}

\author{
Xue Qing \\ Academy of Armored Forces Engineering \\ AAFE \\ Beijing, China \\ xue_qing@yeah.net \\ Deng Qing \\ Academy of Armored Forces Engineering \\ AAFE \\ Beijing, China
}

\author{
Gao Huibo \\ Academy of Armored Forces Engineering \\ AAFE \\ Beijing, China \\ huibode@163.com \\ Li Guo \\ Academy of Armored Forces Engineering \\ AAFE \\ Beijing, China
}

\begin{abstract}
This paper introduces in a limited sense. Based on the virtual soldier, a limited perception of the model framework is built. A virtual model of the soldiers of the limited vision, limited hearing model and the limited memory model are also built.
\end{abstract}

Keywords-Bounded Rationality, Perceived Behavioral Modeling, Virtual Soldiers

\section{INTRODUCTION}

With the rapid development of computer technology, combat simulation system requires more and more high fidelity and reliability. The authenticity and credibility of virtual soldiers' behaviors will have an important impact on the whole system.

The theory of bounded rationality is used to explain differences between the rational person and the real man. Taking the virtual agent and rational person in the similarity of behaviors into account, this article will apply the theory of bounded rationality to the modeling of the perceived behavior of virtual soldiers. This virtual soldier which is added the limited rationality is no longer an omnipotent robot, but has the limited perception like the real person. This improve the credibility of perceived behaviors of virtual soldiers better.

\section{CONCEPTS}

The traditional virtual soldier in combat simulation system is the virtual agent which is controlled by computer. It's easy to interact with the battlefield environment. It can get all the information of all environmental entities from the system[1,2]. It has the information infrastructure which the ordinary people don't have.

Building awareness is the basis of behavioral models[3], and is the initial manifestation of intelligent virtual soldiers. Perception of virtual soldier is that virtual soldiers interact independently with the surrounding virtual environment, and obtain information needed for decision making.

What make virtual soldiers behave as realistically as possible, the virtual soldiers and real soldiers in the perception of perception should be the same limitations. Virtual soldiers should have such a limited perception of rational behavior: get limited environmental information and the faith of uncertain and incomplete perception because of their limited perception. In a virtual battlefield environment, They behave like real people with the characteristics of limited rationality.

\section{THE MODEL OF THE LIMITED PERCEPTION OF VIRTUAL SOLDIERS}

The perception of virtual soldiers are the basis of their making decision[4]. It directly impact on the fidelity and the credibility of virtual soldiers' behavior. Like real soldiers, the virtual soldiers perceived the external environment information with relationship with environmental factors. Limited perception of virtual soldiers is mainly embodied in the limited vision, limited hearing and limited memory. Figure 1 shows the overall structure of the model of virtual soldiers' perception.

A. Limited visual model

Perception of the limited virtual soldiers most directly reflected in the limited vision. Vision model in the limited perception is mainly reflected in two aspects : uncertainty and incomplete information. It is said that the limited virtual soldiers aren't able to perceive information completely and aren't able to perceive the complete information. Establish a realistic model of a limited visual perception in the following seven steps:

(1) Determine the visual range;

(2) Determine the visual range of the soldier' s perception;

(3) Visibility of the objects of perception;

(4) Determine whether the perception is visible;

(5) Determine the level of visual perception;

(6) Memory integrated information;

(7) Form a comprehensive perception.

Identify and determine the visual range. First, determine whether the object is in the soldier's virtual visual range. The scope of the virtual soldier's perception is not a simple sphere, but is a hemisphere. The radius of the hemisphere is D. Therefore, the process can be converted to determine the relationship between the virtual soldier's eyes and the center of the perceived object. Then determine the angle between the direction of projection and eyes. If less than 90 degrees, the object is in the visual perception, or not in the scope of their visual perception. 
The visual factors are based on the limited perception. When virtual soldiers are in the process of visual perception, there are many objective and subjective factors which would affect the results observed. For example: the distance between the object perceived, the volume size, the speed of the object, the visibility of the environment and so on. But considering all of the content will make the system speed slowly. So we usually choose a few factors which is more important.

Determine the level of visual perception. According to the different performance of seeing, the perception of the object in this paper is divided into three levels : the level of detection $\mathrm{Ld}$, the level of recognition $\mathrm{Li}$, the level of understanding Lc. The level of perception is the lowest layer. Soldiers can only get their perception of this object. During the level of recognition soldiers can identify some attributes of the object. During the level of understanding, soldiers can perceive attributes which they are interesting in. Determining the level of visual perception is determined by the visibility $\delta$ : When $0 \leq \delta<0.3$, the level of detection Ld ; When $0.3 \leq \delta<0.7$, the level of recognition $\mathrm{Li}$; When $0.7 \leq \delta \leq 1$, the level of understanding Lc.

Form a comprehensive perception. Determining the perceptual level, virtual soldiers form an initial perception. The comprehensive perception is formed by five groups.Message $1=<\mathrm{ID}$, object, attribute, visibility, grade $>$. ID is a temporary number. 'object' is the name of the object. 'attribute' is the attribute of the object. 'visibility' is the visibility of the object. 'grade' means the level of the perception.

\section{B. Limited auditory model}

Virtual soldier's limited hearing is mainly dependent on its limited scope and limited hearing ability to distinguish sounds. The virtual soldier hears the sound by setting the. Trigger. When the sound triggers a trigger, virtual soldier within the radius of the sphere of it gets a voice message. The message includes ID, location of the source and attributes of the sound such as intensity, frequency and so on. Soldiers under the current task and sound distance to determine whether to notify this information and change the direction of visual perception to the voice of the region.

$$
\text { Sound }_{i}=k_{s} \times \text { sound } / d
$$

Sound $i$ is the strength of the voice that virtual soldier's perceived. sound is the strength of the voice. $d$ is the distance between sound occurred and the virtual soldier. ${ }^{k}$ s is the auditory factor.

The process of modeling virtual auditory perception is shown in Figure 4. It is divided into six steps:

(1) Determine the scope of sound source transmission;

(2) Determine the strength of the voice that virtual soldier's perceived and judge whether it is over the intensity;

(3) Determine the frequency of the sound source is in or out of the range of virtual soldier's hearing;

(4) Determine whether there is masking effect;
(5) Get the preliminary auditory perception;

(6) Form a comprehensive perception.

\section{Limited memory model}

Perceptual model of the memory model plays the role of buffer $[5,6]$. When the memory of the memory model is full, you must get some of the memories out from the store information. Such elimination mechanism is similar to the phenomenon of human memory. Therefore, using the replacement strategy must also reflect some characteristics of forgotten memories. Include: (1) Some important information is difficult to be forgotten; (2) The earlier memory is more easily forgotten. Because of the above characteristics, we use circular queue to store the information in the memory model. The storage memory model is shown in Figure 5. When visual and auditory perception through the filter device, the object in the battlefield environment is considered as the perceived object. The memory model stores the corresponding information in the current available space in the circular queue. The information includes six parts: Perception $=<\mathrm{ID}$, sensor, object, attribute, precision, term>

ID, where the information stored in the memory mode. The ID is unique. Through this identity can easily obtain the general information about the object. Sensor, the information is perceived by. Attribute, some attribute information of the perception. Precision, the accuracy of the information. Term, time and priority of the memory information. According to the stability and the importance of perception, the time is decided. When the memory is full, the current pointer is pointing ' $n$ '. the memory model will compare the information in the storage and the record ' $n$ '. If the term of the record is larger, it is compared with ' $n+1$ ' until the same or less term is found and replace it. Since the storage ring structure of the queue, the earlier information of the memory in the same priority is always got out. So most of the observed objects are only in the memory storage for a limited time. After a period of time, they will be removed like characteristics of the forgotten memory.

\section{CONCLUSION}

The paper introduces the virtual soldiers in a limited sense, builds a framework of the limited perception model, designs the limited visual model, the auditory model and the limited memory model. It is hoped that they can provide some help in building the model of virtual soldiers

\section{REFERENCES}

[1] A, L. The evolution and simulation of a decision maker [M] Analysis of Motivational Processes, ed.London: Totaes F and Halliday Academic Press, 19802 Multigen-Paradigm Inc.Creating Models for Simulations [M]: Multigen Paradigm Inc, 2003

[2] Multigen-Paradigm Inc.Creating Models for Simulations [M]: Multigen Paradigm Inc, 2003

[3] Zhu Chengcheng Research on Visualization in Urban Battlefield and Application [D]. PLA Information Engineering University, Master Thesis, 2007

[4] Guo Qisheng Dong Zhiming. Simulation of Battlefield Environment [M]. Beijing: National Defence Industry Press, 2005 
[5] R, CMOn Grasp Choice, Grasp Model, and the Design of Hands for Manufacturing Tasks [J]. IEEE Transactions on Robotics and Automation, 1999,5

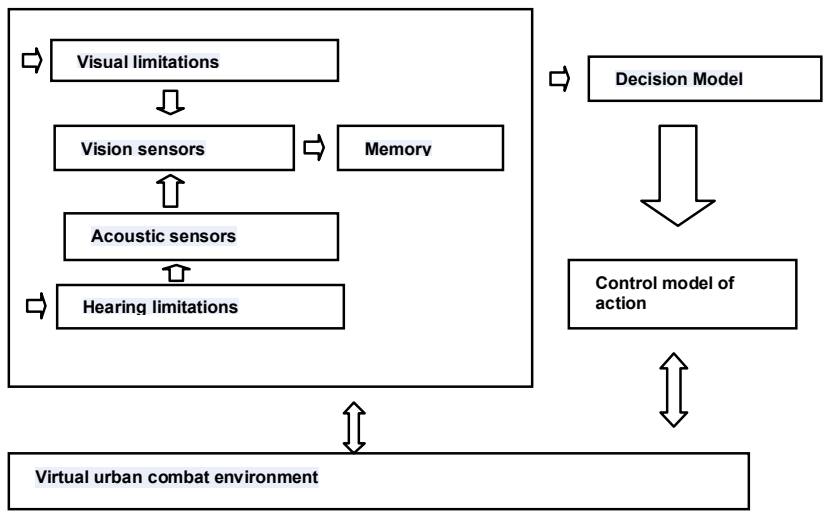

Figure 1. the overall structure of the model of virtual soldiers' perception

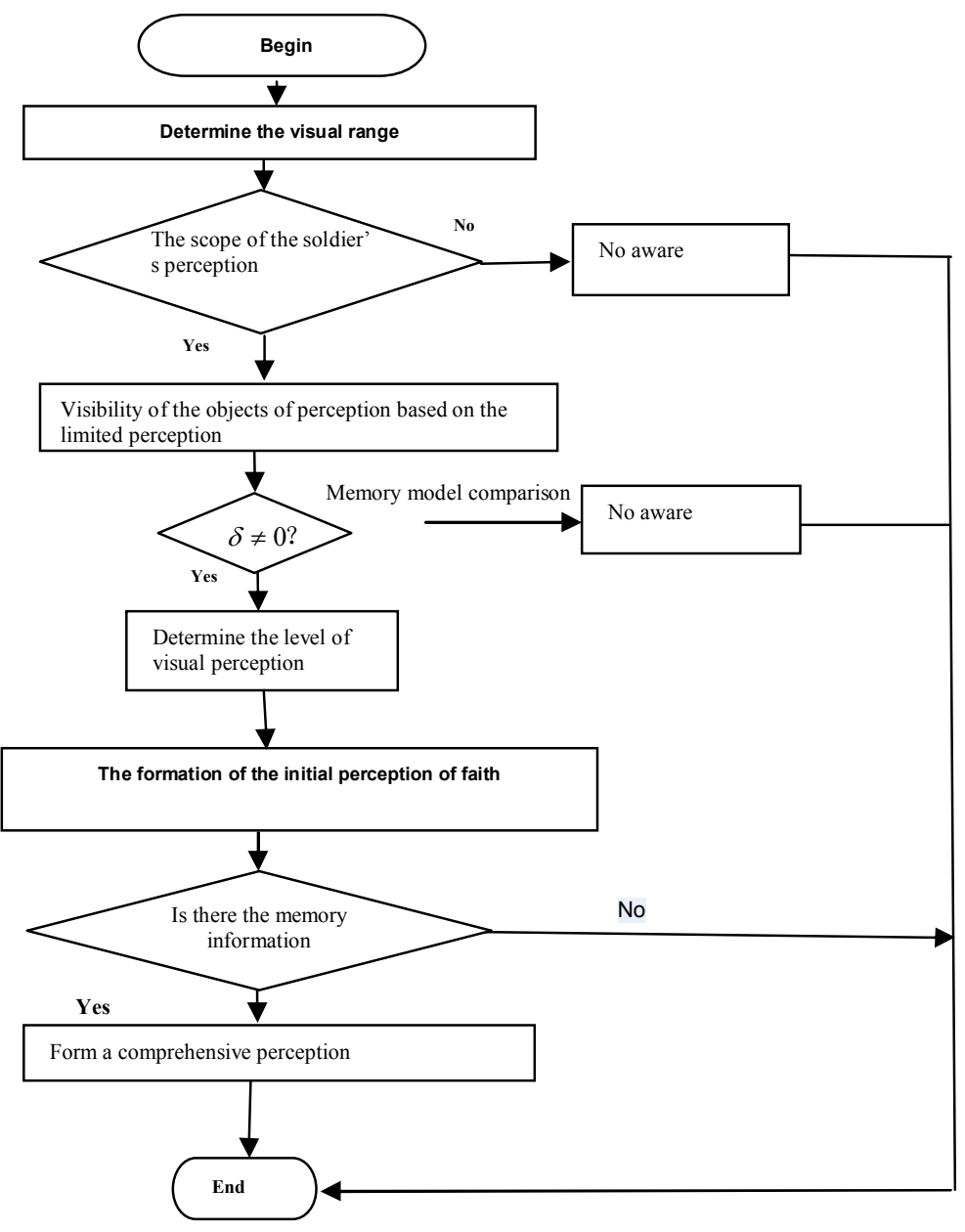

Figure 2. the process of the limited visual perception
6] [6] Karen A. Harper, SSHGLZIntelligent Hostile Urban Threat Agents for MOUT operations [C]. Proceeding of the 9 'th Conference on computer Generated Forces and Behavioral Representation, 2000 


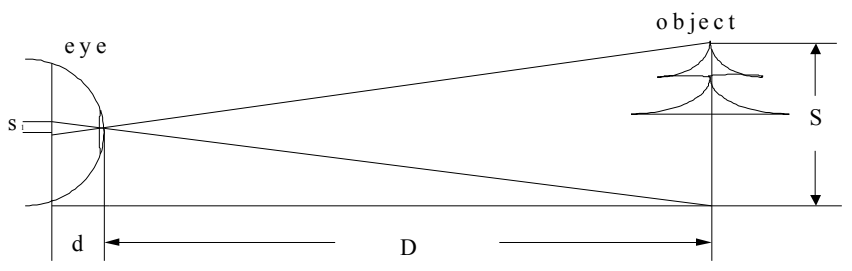

Figure 3. the principle of the visual sense

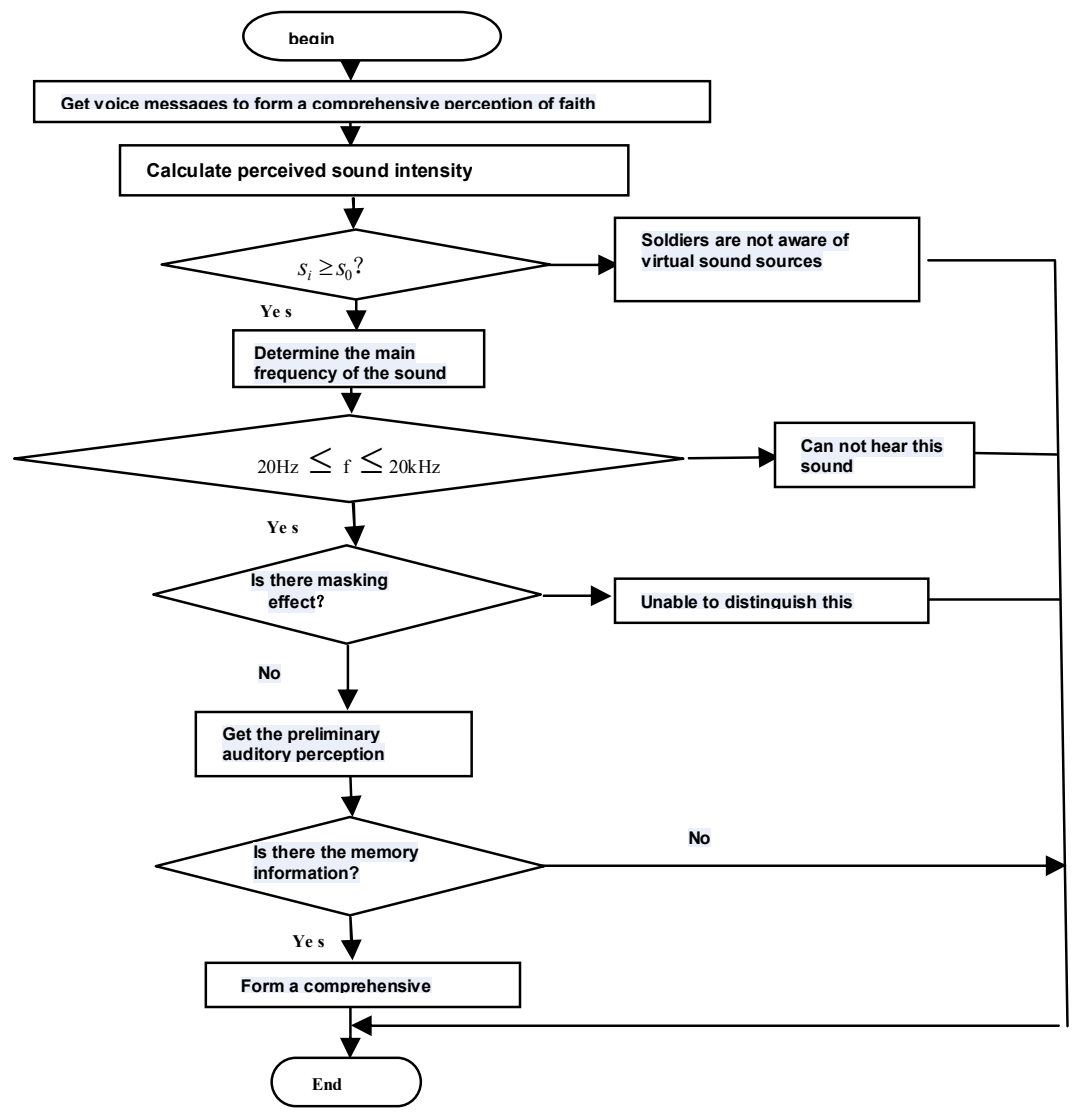

Figure 4. the process of limited auditory perception

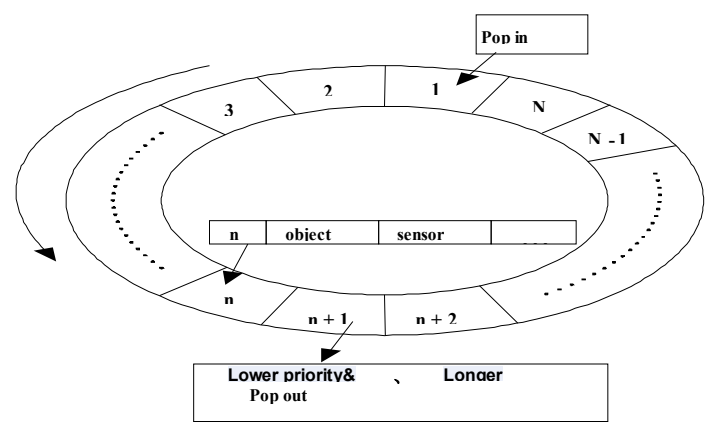

Figure 5. The queue memory storage ring

Published by Atlantis Press, Paris, France.

(C) the authors 